Review

\title{
Uses of Dendrimers for DNA Microarrays
}

\author{
Anne-Marie Caminade *, Clément Padié, Régis Laurent, Alexandrine Maraval and Jean-Pierre \\ Majoral *
}

Laboratoire de Chimie de Coordination CNRS, 205 route de Narbonne, 31077 Toulouse Cedex 4, France

E-mail: caminade@lcc-toulouse.fr. E-mail: padie@lcc-toulouse.fr. E-mail: rlaurent@lcc-toulouse.fr. E-mail:maraval@lcc-toulouse.fr.E-mail:majoral@lcc-toulouse.fr

* Author to whom correspondence should be addressed.

Received: 27 April 2006 / Accepted: 27 July 2006 / Published: 24 August 2006

\begin{abstract}
Biosensors such as DNA microarrays and microchips are gaining an increasing importance in medicinal, forensic, and environmental analyses. Such devices are based on the detection of supramolecular interactions called hybridizations that occur between complementary oligonucleotides, one linked to a solid surface (the probe), and the other one to be analyzed (the target). This paper focuses on the improvements that hyperbranched and perfectly defined nanomolecules called dendrimers can provide to this methodology. Two main uses of dendrimers for such purpose have been described up to now; either the dendrimer is used as linker between the solid surface and the probe oligonucleotide, or the dendrimer is used as a multilabeled entity linked to the target oligonucleotide. In the first case the dendrimer generally induces a higher loading of probes and an easier hybridization, due to moving away the solid phase. In the second case the high number of localized labels (generally fluorescent) induces an increased sensitivity, allowing the detection of small quantities of biological entities.
\end{abstract}

Keywords: dendrimers, microarrays, fluorescence, DNA, dendrons

\section{Introduction}

The growing demand for genetic information has stimulated the search for quick and reliable analytical methods. Among them, biosensors such as DNA microarrays and DNA microchips are gaining an increasing importance, in particular in molecular medicine for gene expression studies, for the detection of nucleotide mutations, or for genotyping of individuals, in forensic applications, but 
also in analytical chemistry, applied in particular to the preservation of food safety and environment quality [1-3].

Typical devices consist of two parts: first a nucleic acid (oligonucleotide or PCR (Polymerase Chain Reaction) products) immobilized at discrete positions on surface activated slides and constituting the probe, and second, a sample constituted of a complex mixture of fluorescently labeled nucleic acids, which contains the target. The supramolecular interaction (the hybridization) between the probe and the target is generally quantified by fluorescence using a high resolution scanning laser. The key point is the hybridization, due to the well-known supramolecular phenomenon of bases pairing [4], in which purine bases (Adenine and Guanine) are hydrogen-bonded to complementary pyrimidine bases (Cytosine and Thymine), creating A-T pairs (two hydrogen bonds) and G-C pairs (three hydrogen bonds) (Figure 1).
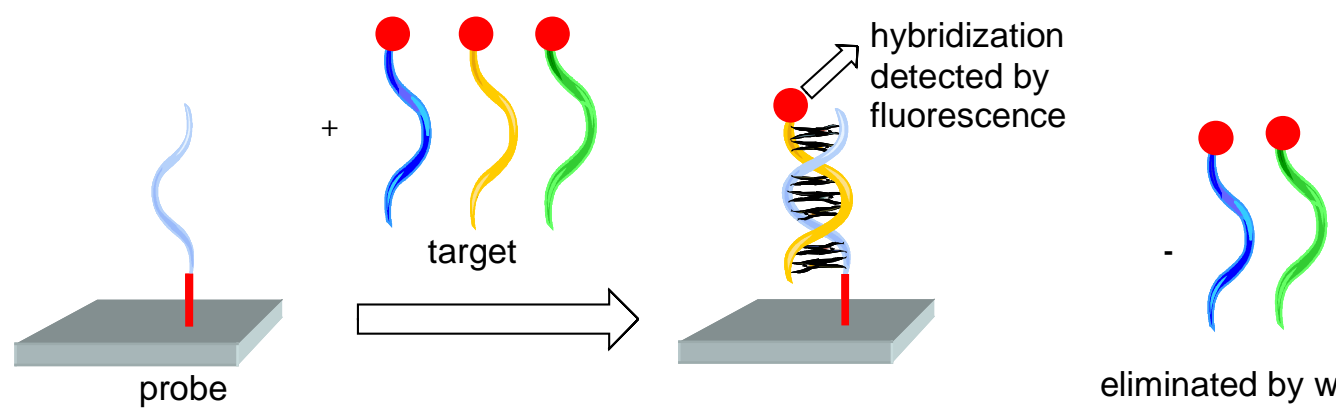

eliminated by washing

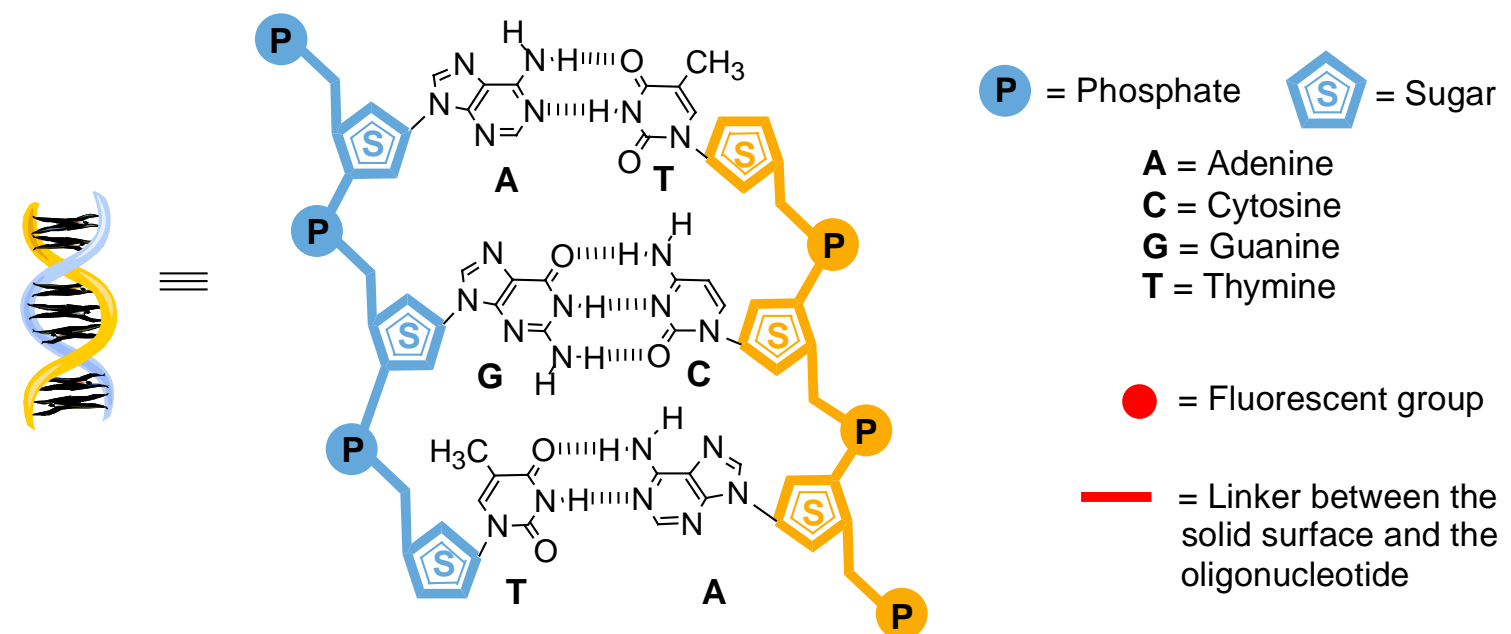

Figure 1. Principle of a DNA chip or DNA array. Only the oligonucleotide complementary to the one immobilized on the surface can hybridize. The hybridization occurs through supramolecular recognition of complementary bases (Adenine with Thymine, and Cytosine with Guanine) linked to the sugar and phosphate backbones, as illustrated by one example.

The degree of sophistication of such devices increases continuously and progresses towards ultrasensitive detection methods. In this short review, we wish to emphasize the role played by dendrimers for improving the sensitivity and reliability of such devices. Dendrimers are macromolecules having a branched structure similar to branches of trees, consisting in repetitive units emanating from a core. Their size depends on the length of the branches and the number of branching levels (the number of "generations"), typically a few nanometers. The shape of dendrimers in solution and for relatively high 
generations approximates a sphere, highly functionalized on the surface. One of the most important features of dendrimers is their numerous end groups, functionalized as desired for particular purposes, and fully accessible (Figure 2). The use of dendrimers in connection with DNA arrays is recent (first paper in 1997), and it is a "hot" topic with $70 \%$ of the papers published within the last three years.

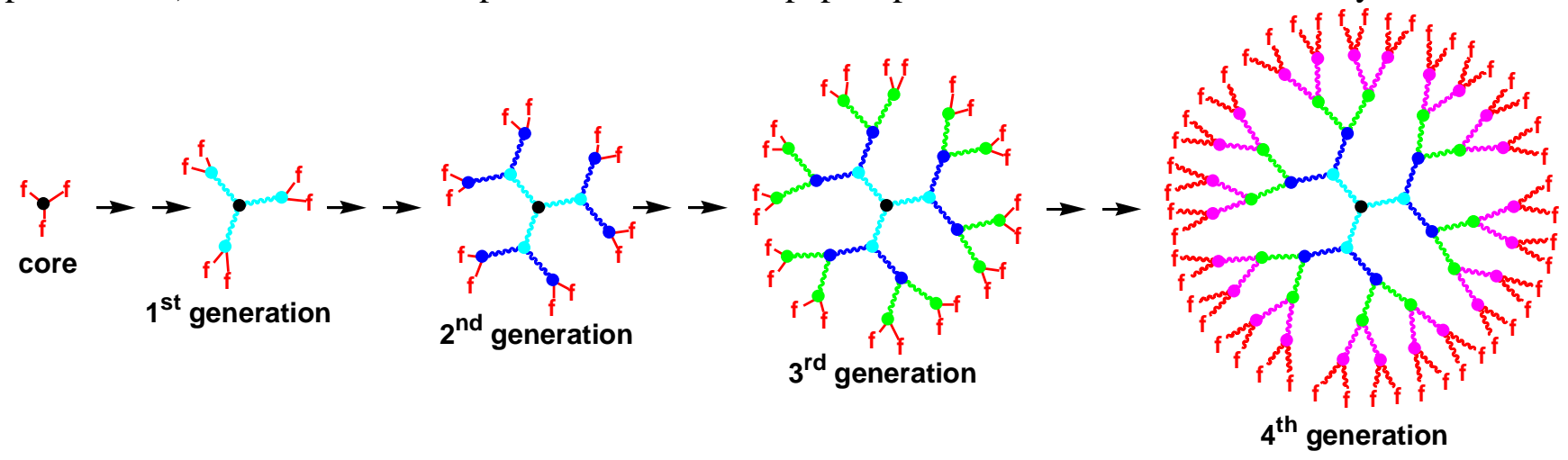

Figure 2. Schematization of the classical method of synthesis of dendrimers, showing the presence of the functional end groups (f).

\section{Role of dendrimers}

Two main types of uses of dendrimers for bioarrays can be distinguished: either the dendrimer is connected to the slide and is used as support of the probe, ensuring the moving of the probe away the solid surface, for improving hybridization, or the dendrimer is used as multiply labeled entities connected to the target for an easier detection. Both topics will constitute the two sub-headings of this review; the first one represents approximately $2 / 3$ of the total number of publications.

\subsection{Use of dendrimers as linkers for the probes to microarray slides}

Glass and silica are typical materials for optical sensors. They have several advantages such as a good chemical resistance and a low intrinsic fluorescence. Their main drawback is the limited density of the surface silanol groups, generally reacted with aminosilane to bind DNA, resulting in a poor surface coverage with DNA. To overcome this problem, substrates affording a higher binding capacity than aminosilane are needed, and dendrimers appear as a particularly interesting alternative, due to their numerous end groups.

The first report connected to this field appeared in 1999, when Beier and Hoheisel [5] described the synthesis of dendritic structures on either aminosilanized glass, or plasma aminated polypropylene surfaces. Acylation reactions using acryloylchloride, followed by the reaction with tetraethylenepentamine, then again with acryloylchloride, and finally with 1,4-bis(3aminopropoxy)butane afford a mixture of branched products linked to the solid surface. These branched products are not true dendrimers due to their inhomogeneous composition, but they possess several $\mathrm{NH}_{2}$ functional groups, which are usable either as starting points for the in situ synthesis of oligonucleotides, or for the immobilization of pre-synthesized oligonucleotides or of PCR products (Figure 3). In both latter cases, the solid phase surface must be activated prior to immobilization, using homobifunctional linking agents, such as phenylenediisothiocyanate (PDITC), disuccinimidyl carbonate, disuccinimidyl oxalate, or dimethylsuberimidate. 


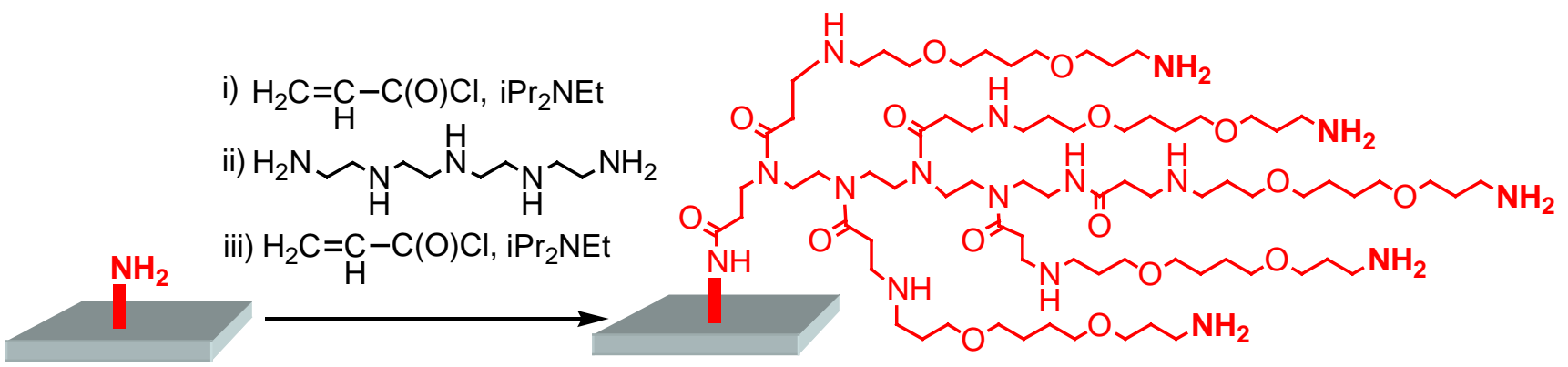

iv) $\mathrm{H}_{2} \mathrm{~N} \sim \mathrm{O}_{\mathrm{O}} \sim \mathrm{NH}_{2}$

Figure 3. Synthesis of a dendritic linker structure, for multiplying the number of reactive site for attachment of nucleic acids. A mixture of dendritic structure is created by this procedure, only one type is shown.

The first example of use of true dendrimers was given by Benters, Niemeyer and Wöhrle [6], who grafted the fourth generation PAMAM (polyamidoamine) dendrimer [7] (64 $\mathrm{NH}_{2}$ groups) onto glass surfaces pre-treated by 3-aminopropyltriethoxysilane and either disuccinimidylglutarate (DSG) or PDITC. After the grafting of PAMAM dendrimers on the surface, the homobifunctional reagents DSG and PDITC were used both to intermolecularly crosslink the dendrimers and to generate reactive groups suitable for reaction with amino-derivatized oligonucleotides (Figure 4). It was expected that the crosslinking should improve the stability of the array. The best results were obtained with the arrays generated using PDITC. In this case, high homogeneity of the spots was observed, as well as a remarkable stability. The intensity of the signal remained constant for more than 110 simulated regeneration cycles, showing that these slides are fully reusable. Thus, these arrays are particularly interesting, as emphasized also by their ability to immobilize proteins [6].
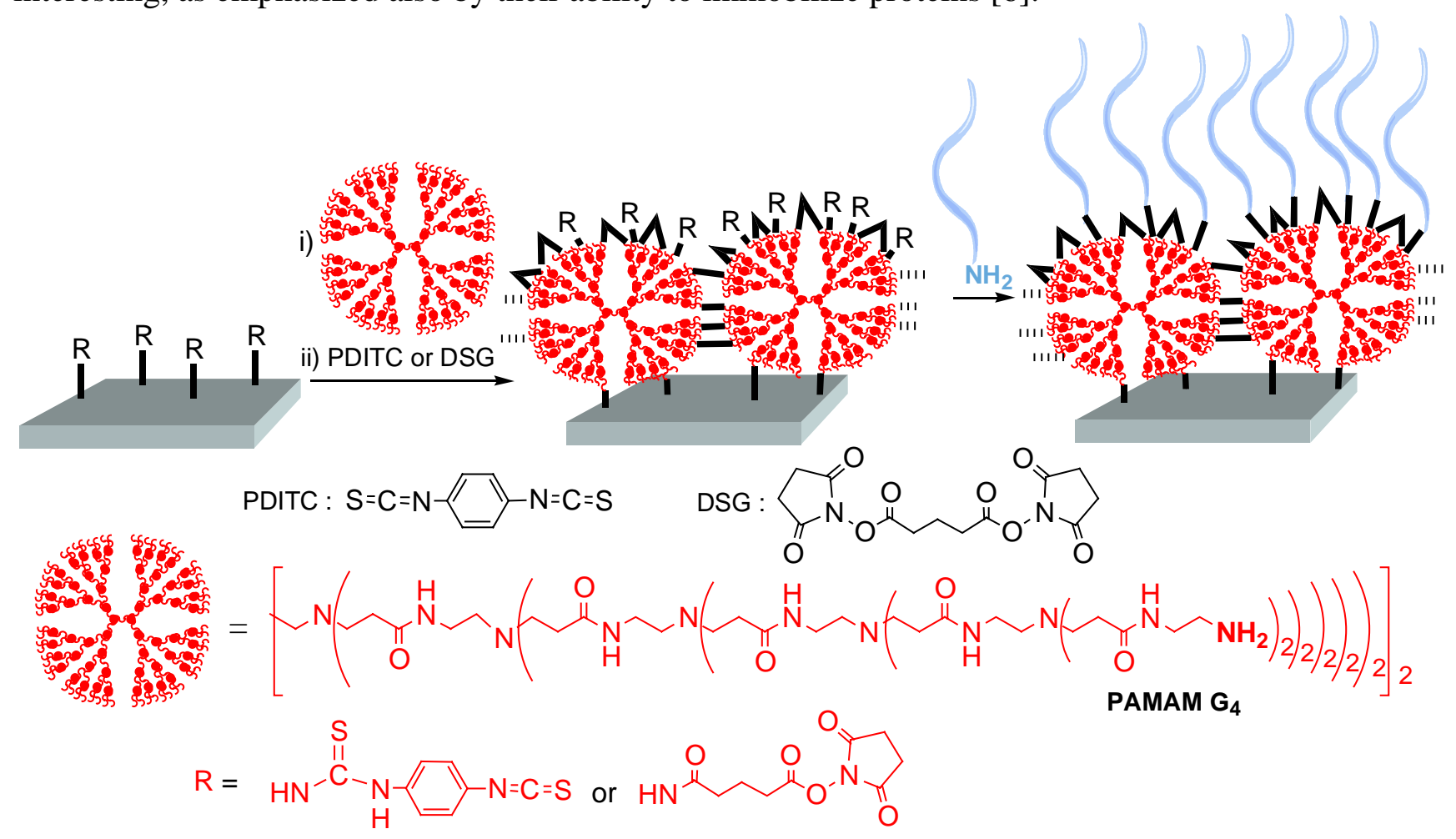

Figure 4. Grafting of PAMAM dendrimers on a surface, then cross-linking and activation using PDITC or DSG, to allow the grafting of amino-modified oligonucleotides. 
A very closely related work was published later, the only difference concerning the type of function linked to the surface of the material. In this case, the R substituents in Figure 4 are aldehydes, able to react in condensation reactions with the $\mathrm{NH}_{2}$ end groups of PAMAM dendrimers, to create imine bonds, reduced by $\mathrm{NaBH}_{4}$ to afford amines, increasing the stability. The grafting of oligonucleotides is carried out as above using PDITC [8].

However, these arrays have a drawback, concerning the loading in oligonucleotides. Only an approximate twofold higher loading is achieved with the dendritic surface compared to classical surfaces, presumably indicating that many functional groups of the PAMAM dendrimers are consumed to form the polymeric network [6]. Thus, it appeared interesting to test the same type of methodology, but avoiding cross-linking [9]. The grafting of the fourth generation PAMAM dendrimer was carried out as already illustrated in Figure 4, starting from an isothiocyanate- a carboxyl- or an epoxymodified surface. Subsequently, glutaric anhydride, N,N'-dicyclohexylcarbodiimide, and Nhydroxysuccinimide were used as activating agents of the $\mathrm{NH}_{2}$ terminal groups, instead of PDITC and DSG, avoiding cross-linking. Then, amino-modified oligonucleotides were grafted to the dendrimer, and subsequently, hybridization with a complementary Cy5-labeled oligonucleotide probe was carried out. The signal intensity shows approximately 10-fold higher fluorescence for the dendritic linker system compared to classical systems (Figure 5). Furthermore, up to 10 regeneration and rehybridization procedures were carried out without loss of signal intensity. Single Nucleotide Polymorphism (SNP), which is particularly important for location of disease-causing genes, are also clearly detectable in hybridization assays. In particular, one mismatch in Cy5-labeled 212mer single stranded DNA obtained by PCR leads to a decrease in signal intensity of 60-92\%, depending on the position of the mismatch.

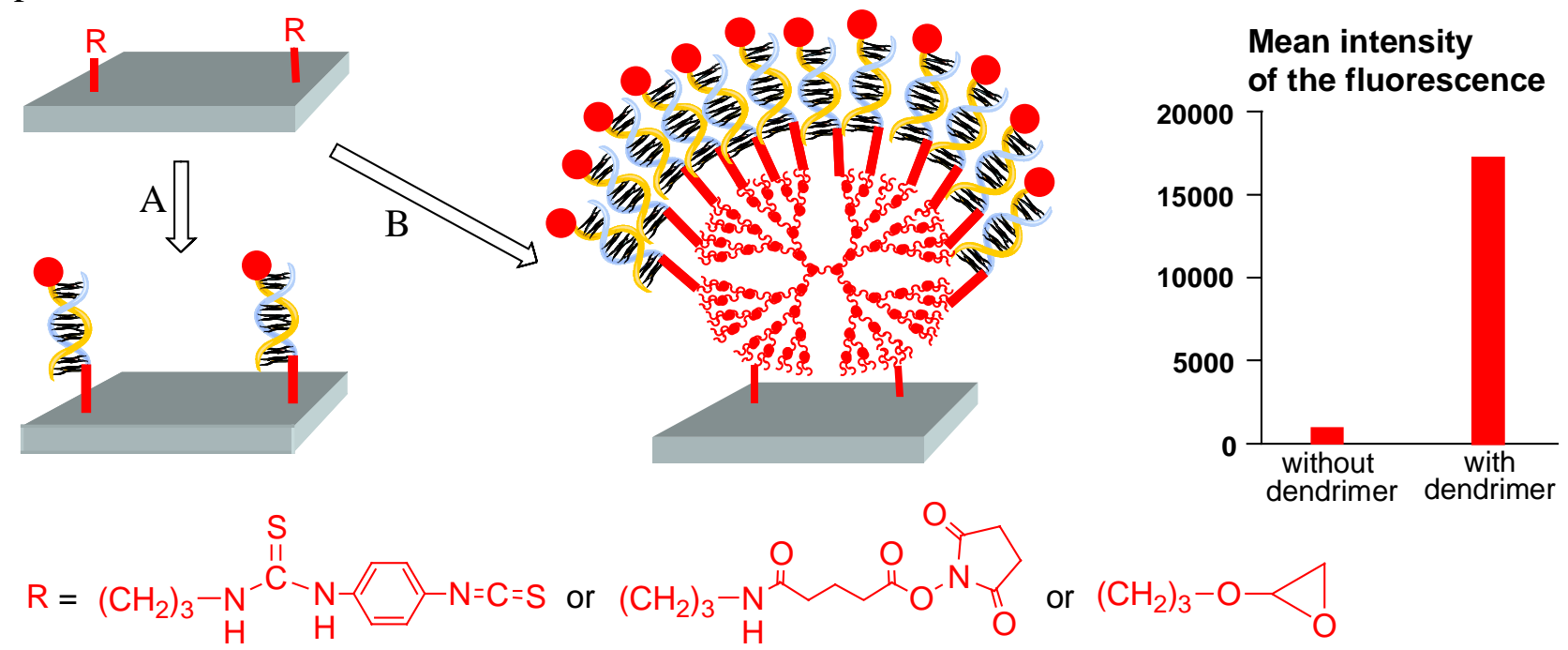

A:

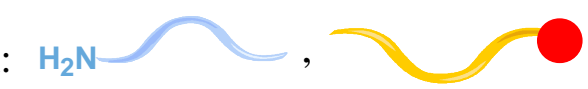

B: PAMAM G

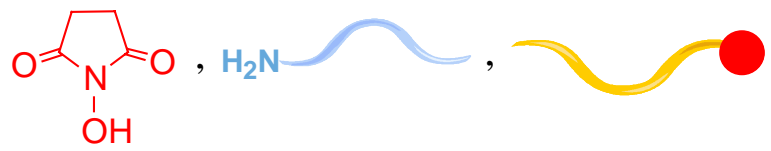

Figure 5. Comparison of the fluorescence intensity measured after hybridization with a fluorescent labeled oligonucleotide on a "classical" surface and on a surface modified by PAMAM dendrimers. 
Other types of dendrimers were used for obtaining DNA microarrays. The most closely related to PAMAM dendrimers are DAB-AM dendrimers (poly(propyleneimine) dendrimers) [10], possessing $\mathrm{NH}_{2}$ end groups. The experiment was carried out with generation 3, possessing $16 \mathrm{NH}_{2}$ end groups, and the epoxide modified solid surface shown in Figure 5. Oligonucleotides and cDNA were noncovalently immobilized on this dendritic surface. A schematic illustration of the supramolecular interaction between DNA and the functional groups of DAB-AM is illustrated in Figure 6. However the results obtained for hybridization and fluorescence with these dendrimer-modified slides are not better than the results obtained for non-dendritic arrays [11].
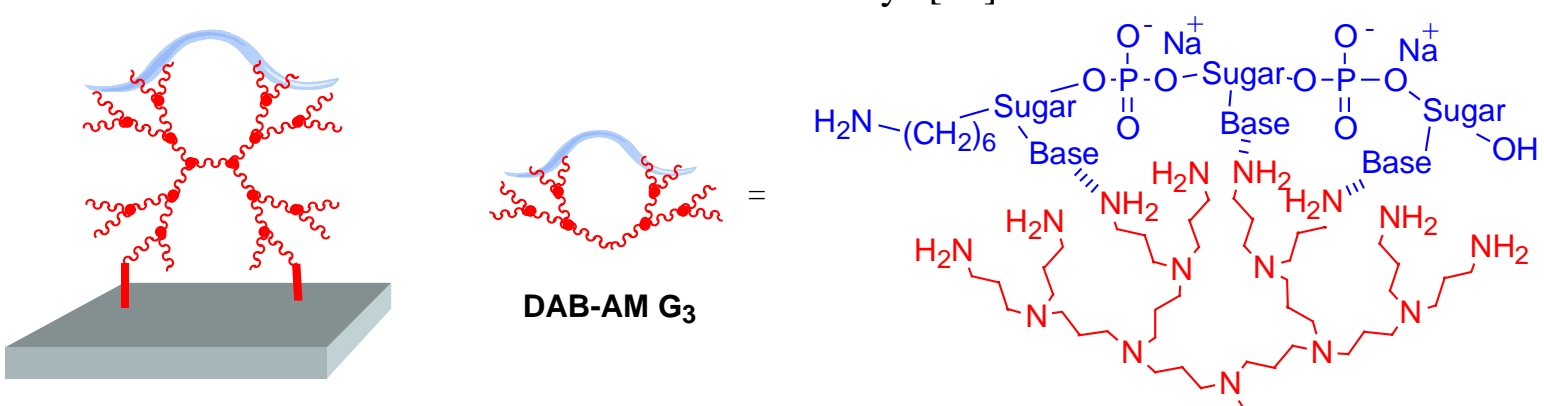

Figure 6. Presumed type of supramolecular interaction between an oligonucleotide and the end groups of DAB-AM dendrimers.

Our contribution to the field of dendritic bioarrays began in 1999, with the immobilization of a protein (Human Serum Albumin (HSA)) [12]. A fifth generation phosphorus-containing dendrimers built from a trifunctional core and bearing aldehyde end groups $\left(\mathbf{G}_{5}\right)$ was added to quartz or glass slides classically pre-treated by aminopropyltriethoxysilane [13]. AFM (Atomic Force Microscopy) images of the slides showed a total coverage of the surface by the dendrimers covalently linked to the surface by imine linkages. Immobilization of proteins was attempted, expecting that it could be accomplished by reaction of the aldehyde groups of the dendrimers with the $\mathrm{NH}_{2}$ groups of proteins. HSA was chosen as a model protein. On the AFM picture shown in Figure 7, the presence of clusters of HSA can be detected, confirming the immobilization.

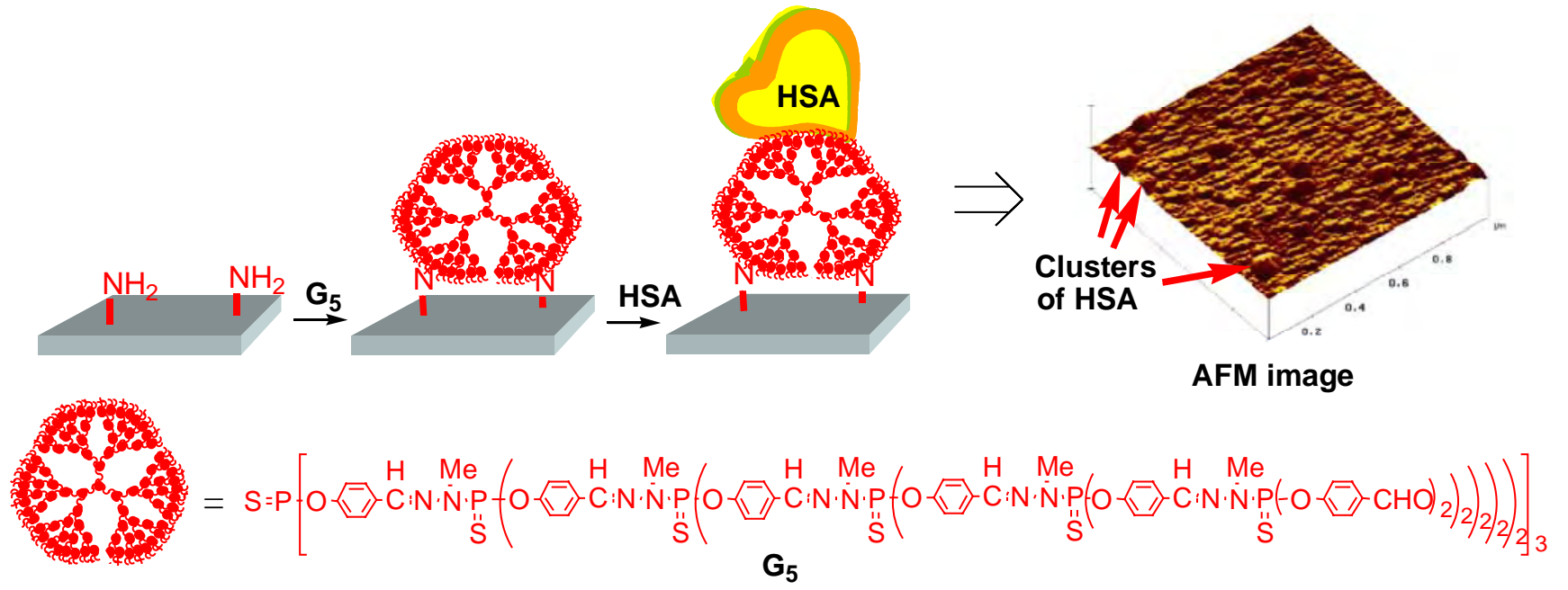

Figure 7. Structure of the fifth generation phosphorus dendrimer built from a trifunctional core and bearing 96 aldehyde end groups, its reaction with a slide functionalized by $\mathrm{NH}_{2}$ groups, and the subsequent immobilization of Human Serum Albumin, analyzed by AFM. 
These first experiments showed that phosphorus dendrimers possessing aldehyde end groups can lead very easily to the elaboration of DNA microarrays. Indeed, the aldehydes allow both the direct grafting to the $\mathrm{NH}_{2}$ groups of the solid surface of the slide, and also the direct reaction of the remaining aldehydes with amino-modified oligonucleotides, without any additional linker for both reactions, contrarily to all the previously reported examples. Analogous phosphorus dendrimers, built from a hexafunctional (cyclotriphosphazene) core instead of a trifunctional core was used (Gc); this core allows to multiply by two the number of end groups at the same generation, compared to previous core (G). Generations 1 ( $\mathbf{G c}_{\mathbf{1}}, 12 \mathrm{CHO}$ end groups) to 7 ( $\mathbf{G c}_{\mathbf{7}}, 768 \mathrm{CHO}$ end groups) [14] were tested for the elaboration of DNA microarrays, using a 35mer DNA probe to be hybridized with a 15mer Cy5-labeled complementary strand. The best results concerning the signal-to-noise ratio were obtained with generations 4 to 7 , thus generation $4\left(\mathbf{G c}_{4}\right)$ was used, because it is easier to synthesize than higher generations. The immobilization efficiency of this "dendrislide" was tested with different concentrations of an amino-modified 35mer oligonucleotide Cy5-labeled, and compared to 10 commercially available glass slides. The fluorescence intensity reaches saturation at $10 \mu \mathrm{M}$ DNA probes, as well as for two others slides. Thus, the binding efficiency of the dendrislides is good, in particular when compared to aminosilanized glass slides, but not better than some commercially available slides [15]. However, this result does not preclude the efficiency of the target capture (the hybridization), especially at low concentration.

To quantify the target/probe hybridization sensitivity, "dendrislides" and 12 commercially available glass slides were functionalized by a 35mer oligonucleotide, spotted at $10 \mu \mathrm{M}$ in solution then hybridized with increasing concentrations of a Cy5-labeled 15mer oligonucleotide complementary to the probe, from $0.001 \mathrm{nM}$ to $100 \mathrm{nM}$. The logarithmic expression of signal intensities versus concentration of targets illustrates a clear advantage of the dendrislides over all other microarrays at target concentrations $<1 \mathrm{nM}$. At $0.001 \mathrm{nM}$ of DNA target a fluorescence signal was still quantifiable only using the dendrislides (Figure 8) [15]. Thus, the detection sensitivity of dendrislides is 10- to 100fold higher than arrays made with most other functionalized glass slides. This high hybridization sensitivity is particularly interesting for studies involving very low amounts of biological materials.

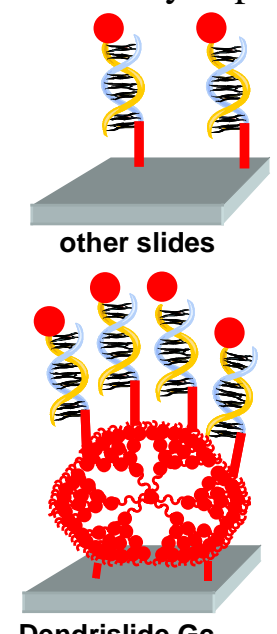

Dendrislide $\mathrm{Gc}_{4}$
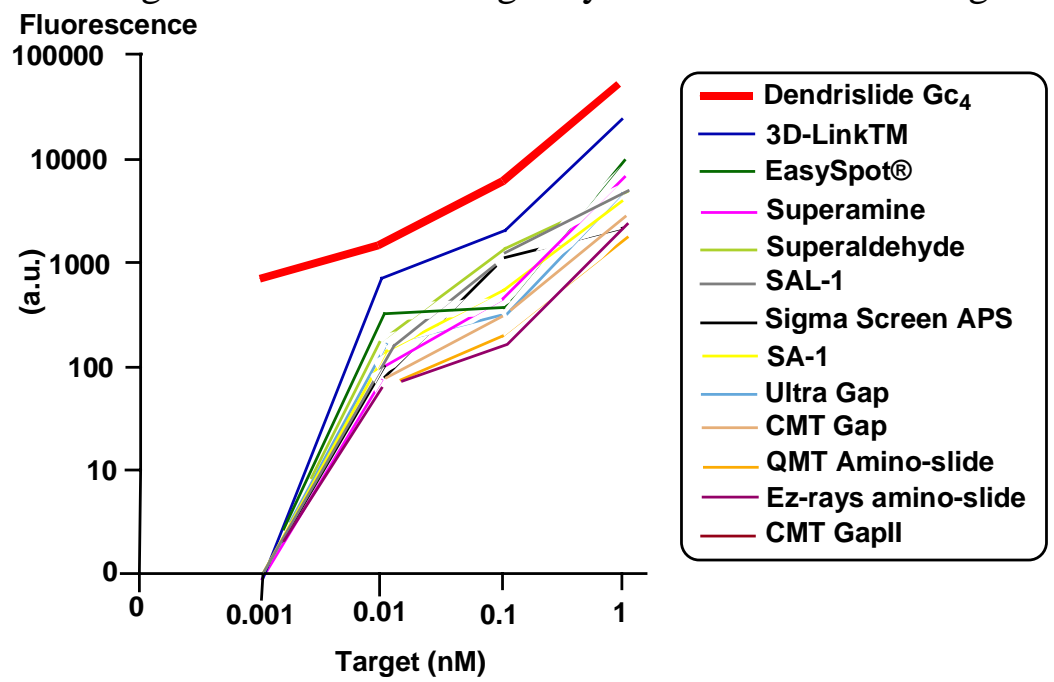

Figure 8. Measurement of the hybridization efficiency of the "dendrislide" elaborated from $\mathbf{G c}_{\mathbf{4}}$, compared to the efficiency of 12 commercially available slides. A fluorescence signal is detectable only with the dendrislide at $1 \mathrm{pM}$ concentration of target. 
The reusability of the dendrislides was also tested and was found excellent even after 10 hybridization/stripping cycles in the case of oligonucleotides [16], but the stripping process was found less effective using dendritic arrays bearing the whole yeast genome spotted as PCR products [15]. Interestingly, single nucleotide polymorphism (SNP) can be detected with the dendrislides. The hybridization of four 15mer oligonucleotides having a single base mutation in the middle (position 8) with a 35mer oligonucleotide probe grafted on the dendrislide is only effective with the oligonucleotide sequence strictly complementary [16]. An alternative method to deposit the complementary oligonucleotide onto the dendrislide has been proposed. It consists in microcontact printing using stamps, the "ink" being the oligonucleotide to be deposited. Comparison of the quality of the printing on the dendrislide and on a classical slide by fluorescence and by Atomic Force Microscopy revealed an excellent definition of the contours of the patterns only with the dendrislide [17].

These DNA chips elaborated from the $\mathbf{G} \mathbf{c}_{4}$ dendrimer can be converted to nanocapsule arrays, by grafting on liposomes oligonucleotides complementary to the oligonucleotides bound to the array. Two series of liposomes were synthesized, each series bearing one type of oligonucleotide. A fluorescent dye derived from rhodamine (green) was used for labeling the liposome membrane of one series, and a fluorescent dye derived from Cy5 (red) was encapsulated in the liposome internal volume of the other series. Both series of liposomes were then mixed and deposited on a glass slide spotted with 3 different oligonucleotides; the first one is complementary to one series of liposomes, the second one is complementary to the other series of liposomes, and the third one is not complementary. Following hybridization, detection of fluorescence on the chip revealed colored spots corresponding to the fluorescent dyes used; each kind of liposome binds specifically to the spot corresponding to the complementary oligonucleotide on the chip (Figure 9). Such a process is potentially usable for the encapsulation and spotting of proteins, keeping their conformation and activity within the liposomes [18].

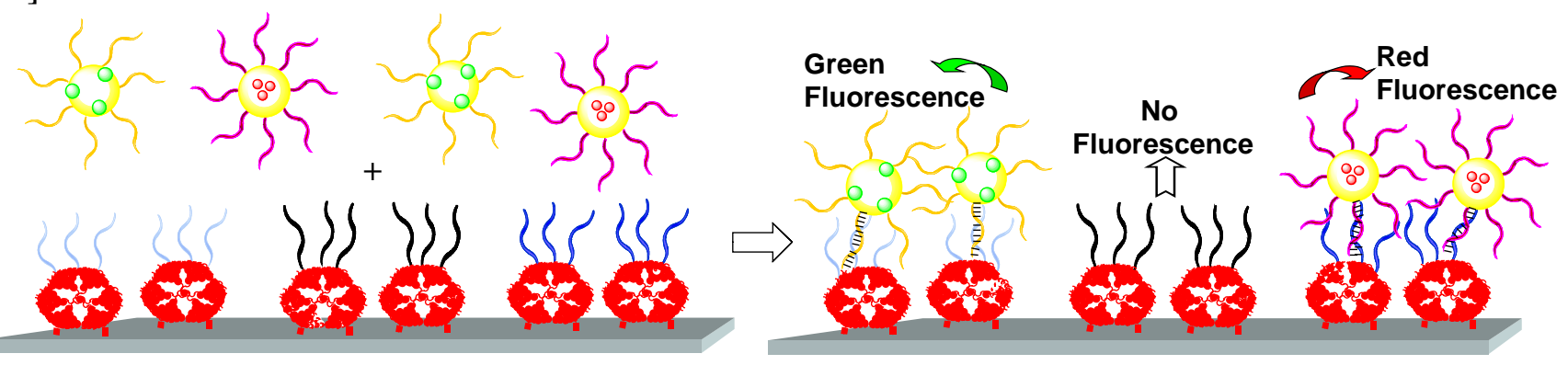

Figure 9. Specific liposome attachment to the array constituted of $\mathbf{G c}_{\mathbf{4}}$ dendrimer via complementary oligonucleotide hybridization; attachment is detected by fluorescence.

The nature of the support of the array can be varied in order to use other methods of detection than fluorescence. The fourth generation phosphorus dendrimer $\mathbf{G}_{\mathbf{4}}$ was linked as previously to a special silica membrane, which is a piezoelectric membrane. The complementary oligonucleotide hybridized in this case does not bear a fluorescent label but a biotin label, well known to be selectively recognized by streptavidin. This functionalized piezoelectric membrane was integrated in a flow injection analysis system, and its resonant frequencies were measured using the optical beam deflection technique. Measurements were carried out on the biotinylated DNA hybridized membrane, and after injection of a 
solution of streptavidin-conjugated gold nanoparticles. The mass loading induced by the supramolecular recognition between streptavidin and biotin is detected by a modification of the resonant frequency of the membrane. The mass sensitivity has been estimated to $-3.6 \mathrm{~Hz} / \mathrm{pg}$, which is by a factor of several hundreds better than state-of-art values for piezoelectric mass-sensing devices; a $3.9 \mathrm{kHz}$ shift of the resonant frequency has been recorded for the membrane without and with colloids (Figure 10), demonstrating the potential use of such devices as biosensors [19].
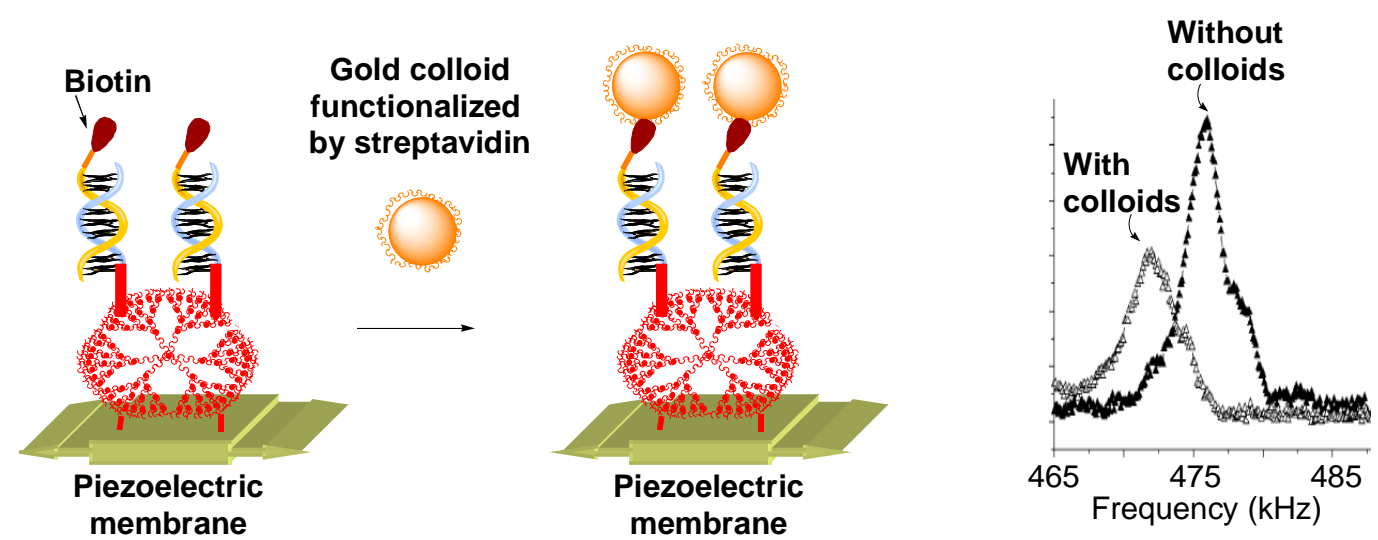

Figure 10. Left: schematic representation of biotinylated oligonucleotides hybridized to complementary oligonucleotides linked to a piezoelectric membrane through the $\mathbf{G c}_{\mathbf{4}}$ phosphorus dendrimer; supramolecular recognition of streptavidin-conjugated gold colloid by biotin. Right: resonant frequencies measured before and after the grafting of gold colloids to the piezoelectric membrane.

Besides "classical" dendrimers, DNA dendrimers were also used for the elaboration of DNA biosensors. These DNA dendrimers are built from dendritic monomers constituted of single-stranded nucleic acid molecules partially hybridized, which can in turn be sequentially hybridized in order to form nucleic acid dendrimers, with a controlled exponential growth governed by supramolecular interactions [20]. About 18 layers of the fourth generation of such DNA dendrimers, possessing as end groups about 30 single-stranded arms specific to the waterborne pathogen Cryptosporidium parvum, were immobilized onto a quartz-crystal microbalance. The numerous probes on the outermost layer accessible to the Cryptosporidium DNA target yielded a three-dimensional surface hybridization and consequently a large resonant-frequency response [21] (Figure 11).

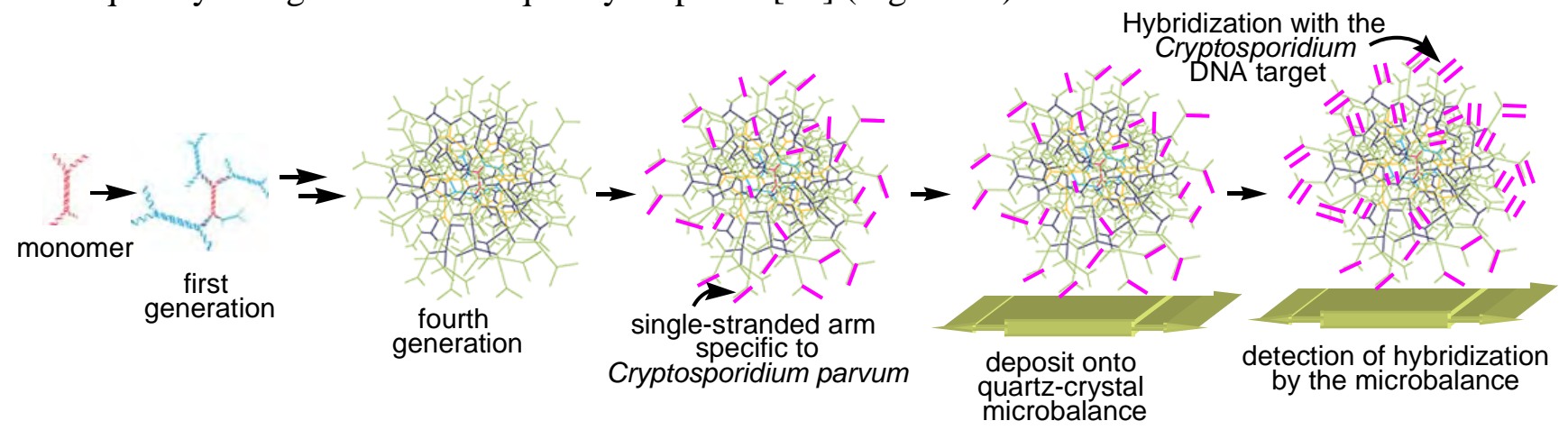

Figure 11. Synthesis of DNA dendrimers by selective hybridizations up to the fourth generation, and functionalization of end groups by specific single-stranded arms. Deposit onto quartz-crystal (only a 
monolayer is shown for simplicity), then hybridization by the target and detection by modification of the frequency-time response of quartz-crystal microbalance.

Cone-shaped dendritic molecules called dendrons possess one functional group located at the core, in addition to the functional groups linked to the surface, and they have been used also very recently for the elaboration of DNA microarrays. Dendrons linked to a solid substrate by their surface groups generate mesospacing on the solid. The grafting of gold nanoparticles on each apex of the immobilized dendrons and their detection by AFM give an average separation of $3.2 \mathrm{~nm}$ [22]. Coupling of aminomodified oligonucleotides with the $\mathrm{NH}_{2}$ of the immobilized dendrons, using $\operatorname{di}(\mathrm{N}-$ succinimidyl)carbonate (DSC) as linker affords DNA microarrays. Such process provides each probe DNA with ample space for hybridization with incoming DNAs, resulting in high hybridization yields (80-100\%) (Figure 12). These particular arrays have been used to detect a successful discrimination ratio of 100:<1 between a complementary pair and three internal single-base mismatched pairs [22]. Single nucleotide variations in the exons 5-8 of the p53 gene in genomic DNAs from cancer cell lines were also detected with a high selectivity and sensitivity [23]. A wide range of temperature $\left(37-50^{\circ} \mathrm{C}\right)$ is usable, and in general the hybridization behavior on these arrays is similar to the solution one [24].

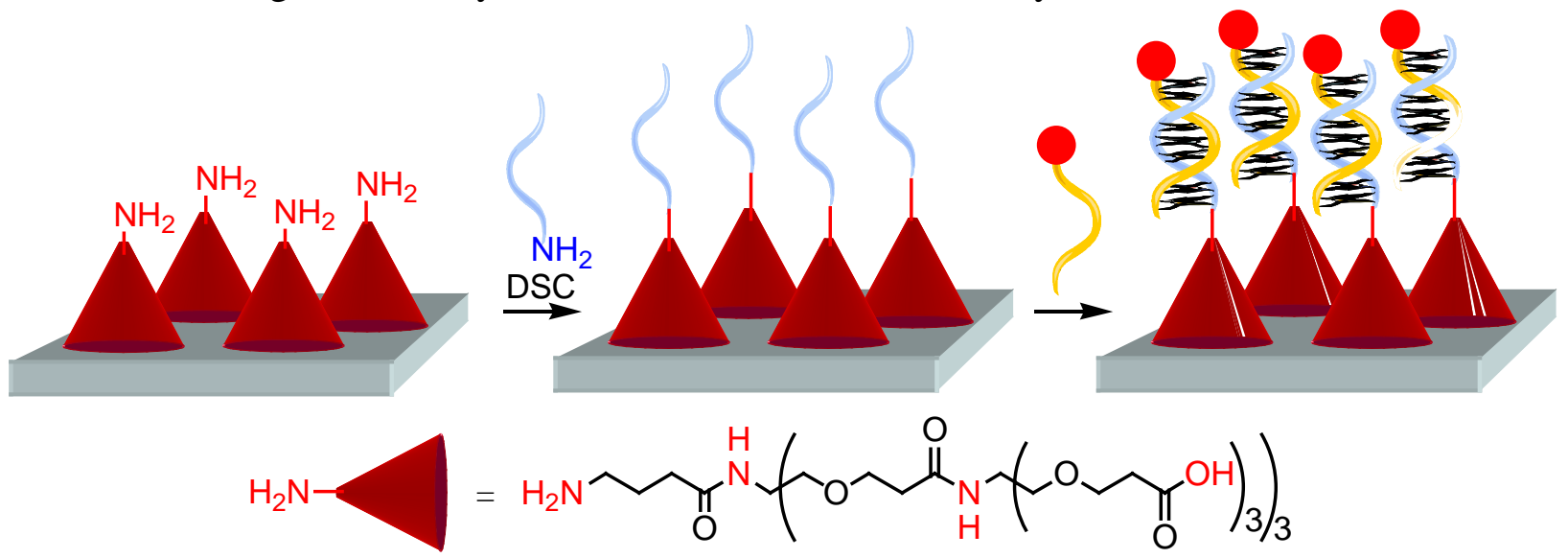

Figure 12. Dendrons grafted to a solid substrate by their surface groups, generating mesospacing; coupling of amine-tethered oligonucleotide through DSC linker. High hybridization yields are obtained with this nanostructured surface.

\subsection{Use of dendrimers as multiply labeled entities connected to the target}

Besides the sensitivity of the slides, which can be improved by using dendrimers as shown in the previous part of this paper, amplification of the signal resulting from detection events can also be enhanced using dendrimers in particular as fluorescent labels. Indeed, the end groups of dendrimers can be fluorescent entities, and their number should afford high fluorescence intensity. However, such highly fluorescent dendrimers must be linked to the target, and dendrons appear as good candidates for such purpose. Indeed, fluorescent dyes can be linked to their end groups, whereas an oligonucleotide or a PCR product can be connected to their core. Such strategies has been applied to various sizes of polyether-type dendrons, bearing Cy3 dyes (1, 2, 34 or 9 dyes) as end groups and PCR amplicons of different human herpes viruses (herpes simplex virus (HSV-1 and HSV-2), varicella zoster virus, Epstein-Barr virus, cytomegalovirus, and HHV-6). The fluorescence signal enhancement via the 
dendrons of the microarrays specifically hybridized to these fluorophore-labeled pathogenic DNAs was up to 30 times compared with a single fluorophore (Figure 13) [25].
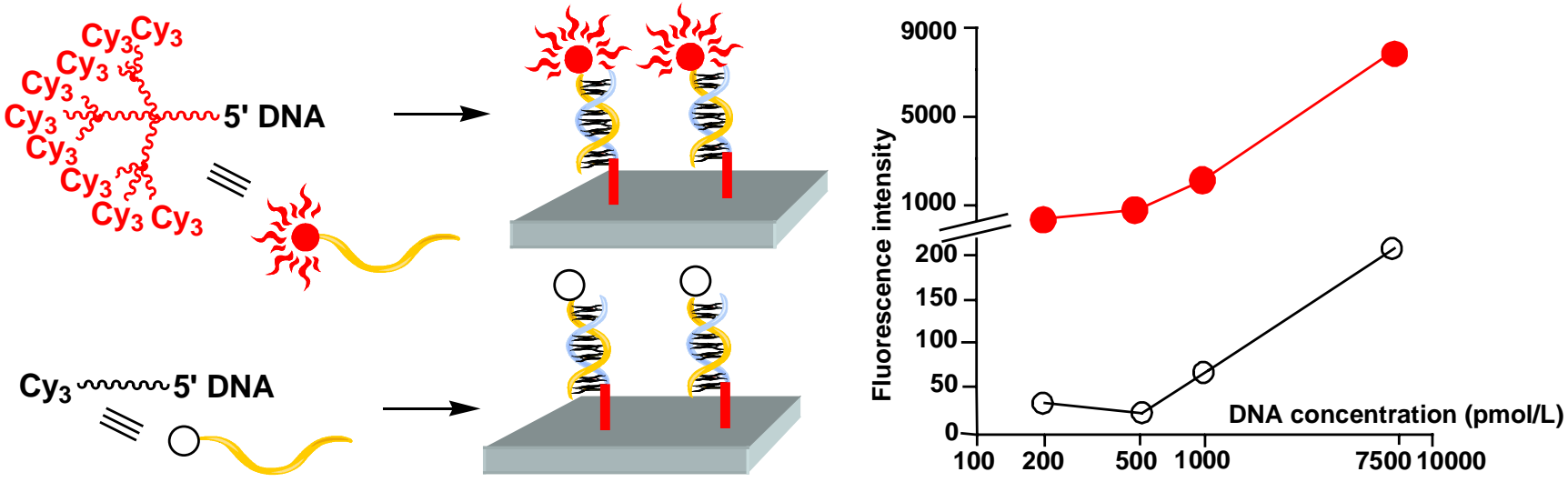

Figure 13. Schematic illustration of a dendron possessing nine Cy3 dyes and one PCR product (for instance HSV-1 amplicon). Fluorescence intensities of the microarray hybridization experiments plotted against the concentration of HSV-1 amplicons, depending on the number of attached fluorophores; white circles: 1 Cy3 per DNA double strand, red circles: 9 Cy3 per DNA double strand.

Besides fluorescence, radioactivity is also a main tool in biology, especially $\gamma_{-}{ }^{32} \mathrm{P}$. The principle shown in Figure 13 for fluorescent entities was also applied to oligonucleotide dendrons [26] containing bunches of nine oligonucleotides labeled with $\left[\gamma_{-}{ }^{32} \mathrm{P}\right] \mathrm{ATP}$, and one specific oligonucleotide linked to the core. These multiply labeled structures showed much higher sensitivity than non dendrimeric (monomeric) labels when used as probes to arrays bearing oligonucleotides complementary to that of the core of the dendron. The large dendrimeric structure does not significantly affect the hybridization yield [27].

Some DNA dendrimers of type shown in Figure 11 have been especially designed to be used as highly fluorescent labels. In fact, the single-stranded arms on the surface of these dendrimers are used to attach two types of functionalities, one type is the label (mainly Cy3, Cy5, Alexa Fluor 546, or Alexa Fluor 647), the other type of functionality will enable the attachment to the desired probe; both are attached by hybridization with oligonucleotides. These types of compounds are called 3DNA ${ }^{\circledR}$ dendrimer by Genisphere Inc. and are generally used for an indirect detection. Indeed, first a 3DNA capture sequence must be linked to the oligonucleotide or PCR products to be analyzed (the target), second the product must be hybridized to the microarray printed with the complementary oligonucleotide or PCR product, and third the fluorescent 3DNA is hybridized with the capture sequence (Figure 14) [28]. This indirect method has been found more efficient than a classical direct method for the labeling of the partial genome of the methylotrophic yeast Hansenula polymorpha and its detection on DNA arrays [29], and for the detection of rare stranscripts available in small quantities, such as hair cells in cochean tissues of mouse ear [30].

These 3DNA dendrimers have been used as highly fluorescent, sensitive and versatile labels for a number of purposes. In particular, they have been used in comparison with more classical techniques for the detection of predetermined ratio values from target external mRNA standards (spikes of Arabidopsis thaliana) in background of total RNA [31], for studying the influence of multiple scanning on the accuracy of detection [32], and for designing a microassay array that quantify the measurement of hybridization stoichiometry in molar units [33]. 


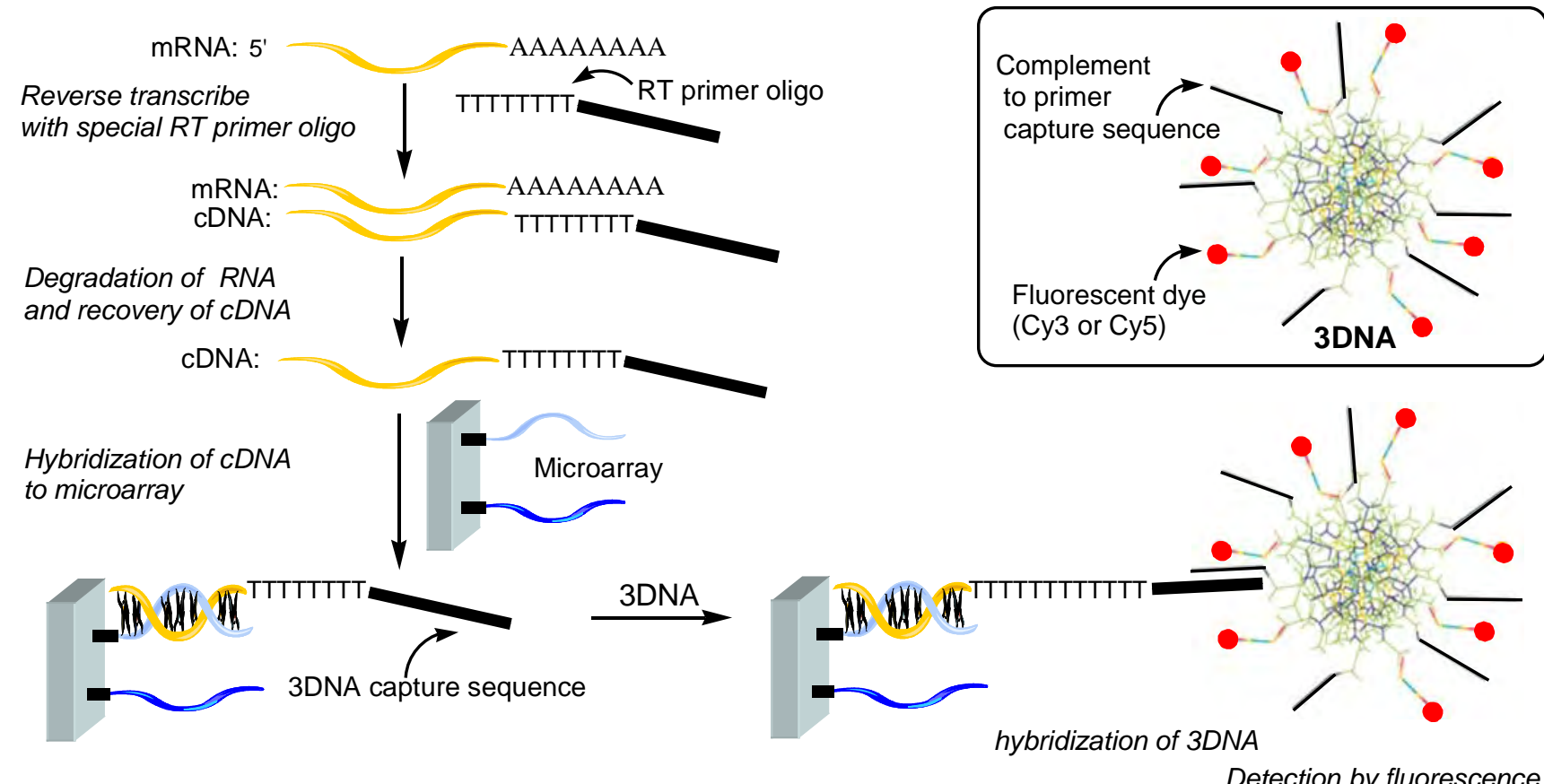

Figure 14. Use of 3DNA dendrimers bearing 45 fluorescent dyes (Cy3, Cy5, Alexa Fluor 546, or Alexa Fluor 647) and oligo/conjugate with application specificity for the indirect labeling of cDNA.

\section{Conclusion}

The use of dendrimers (or dendrons) for improving the sensitivity of microarrays is still in its infancy, however promising leads are already identified. When used as linkers between the solid surface and the oligonucleotide probe, dendrimers generally allow a higher loading in oligonucleotide, and their remoteness from the solid favors hybridization, which occurs as easily as in solution. Furthermore, the systems created using dendrimers are generally very robust and they can undergo without any damage several hybridization / deshybridization cycles. Their reusability has been shown in several cases, for dozens or hundreds of experiments.

When used as fluorescent or radioactive multiply labeled entities linked to the target, dendrimers (or dendrons) are always much easily detected than monomeric labels, inducing important increases in sensitivity, particularly useful when only very small quantities of biological materials have to be analyzed. Such methodology has been applied recently to "suspension microarrays" using beads (microspheres) instead of planar microarrays [34].

\section{References and Notes}

1. Wittmann, C. (Editor) Immobilization of DNA on chips I. Topics Curr. Chem. 2005, 260.

2. Wittmann, C. (Editor) Immobilization of DNA on chips II. Topics Curr. Chem. 2005, 261.

3. Rosi, N.L.; Mirkin, C.A. Nanostructures in biodiagnostics. Chem. Rev. 2005, 105, 1547-1562.

4. Watson, J.D.; Crick, F.H.C. Molecular structure of nucleic acids. A structure of deoxyribose nucleic acid. Nature 1953, 171, 737-738. 
5. Beier, M.; Hoheisel, J.D. Versatile derivatisation of solid support media for covalent bonding on DNA-microchips. Nucleic Acids Res. 1999, 27, 1970-1977.

6. Benters, R.; Niemeyer, C.M.; Wöhrle, D. Dendrimer-activated solid supports for nucleic acid and protein microarrays. ChemBioChem 2001, 2, 686-694.

7. Tomalia, D.A.; Naylor, A.M.; Goddard, W.A. Starburst dendrimers: molecular-level control of size, shape, surface chemistry, topology and flexibility from atoms to macroscopic matter. Angew. Chem. Int. Ed. Engl. 1990, 29, 138-175.

8. Park, J.W.; Jung, Y.; Jung, Y.H.; Seo, J.S.; Lee, Y. Preparation of oligonucleotide arrays with high-density DNA deposition and high hybridization efficiency. Bull. Korean Chem. Soc. 2004, 25, 1667-1670.

9. Benters, R.; Niemeyer, C.M.; Drutschmann, D.; Blohm, B.; Wohrle, D. DNA microarrays with PAMAM dendritic linker systems. Nucleic Acids Res. 2002, 30, e10.

10. de Brabander-van den Berg, E.M.M.; Meijer, E.W. Poly(propylene imine) Dendrimers: LargeScale Synthesis by Hetereogeneously Catalyzed Hydrogenations. Angew. Chem. Int. Ed. Engl. 1993, 32, 1308-1311.

11. Taylor, S.; Smith, S.; Windle, B.; Guiseppi-Elie, A. Impact of surface chemistry and blocking strategies on DNA microarrays. Nucleic Acids Res. 2003, 31, e87.

12. Slomkowski, S.; Miksa, B.; Chehimi, M.M.; Delamar, M.; Cabet-Deliry, E.; Majoral, J.P.; Caminade, A.M. Inorganic-organic systems with tailored properties controlled on molecular, macromolecular and microscopic level. React. Funct. Polym. 1999, 41, 45-57.

13. Launay, N.; Caminade, A.M.; Majoral, J.P. Synthesis and Reactivity of Unusual Phosphorus Dendrimers. An useful divergent growth approach up to the seventh generation. J. Am. Chem. Soc. 1995, 117, 3282-3283.

14. Launay, N.; Caminade, A.M.; Majoral J.P. Synthesis of bowl-shaped dendrimers from generation 1 to generation 8. J. Organomet. Chem. 1997, 529, 51-58.

15. Le Berre, V.; Trevisiol, E.; Dagkessamanskaia, A.; Sokol, S.; Caminade, A.M.; Majoral, J.P.; Meunier, B.; François, J. Dendrimeric coating of glass slides for sensitive DNA microarrays analysis. Nucleic Acids Res. 2003, 31, e88.

16. Trévisiol, E.; Leberre-Anton, V.; Leclaire, J.; Pratviel, G.; Caminade, A.M.; Majoral, J.P.; François, J.M.; Meunier, B. Dendrislides, dendrichips: a simple chemical functionalization of glass slides with phosphorus dendrimers as an effective mean for the preparation of biochips. New J. Chem. 2003, 27, 1713-1719.

17. Thibault, C.; Le Berre, V.; Casimirius, S.; Trévisiol, E.; François, J.; Vieu, C. Direct microcontact printing of oligonucleotides for biochip applications. J. Nanobiotechnol. 2005, 3, 7.

18. Chaize, B.; Nguyen, M.; Ruysschaert, T.; le Berre, V.; Trévisiol, E.; Caminade, A.M.; Majoral, J.P.; Pratviel, G.; Meunier, B.; Winterhalter, M.; Fournier, D. Microstructured liposome array. Bioconjugate Chem. 2006, 17, 245-247.

19. Nicu, L.; Guirardel, M.; Chambosse, F.; Rougerie, P.; Hinh, S.; Trévisiol, E.; François, J.M.; Majoral, J.P.; Caminade, A.M.; Cattan, E.; Bergaud, C. Resonating piezoelectric membranes for microelectromechanically-based bioassay: detection of streptavidin-gold nanoparticles interaction with biotinylated DNA. Sensors and Actuators B: Chemical 2005, 110, 125-136. 
20. Nilsen, T.W.; Grayzel, J.; Prensky, W. Dendritic nucleic acid structures. J. Theor. Biol. 1997, 187, 273-284.

21. Wang, J.; Jiang, M.; Nilsen, T.W.; Getts, R.C. Dendritic nucleic acid probes for DNA biosensors. J. Am. Chem. Soc. 1998, 120, 8281-8282.

22. Hong, B.J.; Oh, S.J.; Youn, T.O.; Kwon, S.H.; Park, J.W. Nanoscale-controlled spacing provides DNA microarrays with the SNP discrimination efficiency in solution phase. Langmuir 2005, 21, 4257-4261.

23. Oh, S.J.; Ju, J.; Kim, B.C.; Ko, E.; Hong, B.J.; Park, J.G.; Park, J.W.; Choi, K.Y. DNA microarrays on a dendron-modified surface improve significantly the detection of single mucleotide variations in the p53 gene. Nucleic Acids Res. 2005, 33, e90.

24. Hong, B.J.; Sunkara, V.; Park, J.W. DNA microarrays on nanoscale-controlled surface. Nucleic Acids Res. 2005, 33, e106.

25. Streibel, H.M.; Birch-Hirschfeld, E.; Egerer, R.; Foldes-Papp, Z.; Tilz, G.P.; Stelzner, A. Enhancing sensitivity of human herpes virus diagnosis with DNA microarrays using dendrimers. Exp. Mol. Pathol. 2004, 77, 89-97.

26. Shchepinov, M.S.; Mir, K.U.; Elder, J.K.; Frank-Kamenetskii, M.D.; Southern, E.M. Oligonucleotide dendrimers: stable nano-structures. Nucleic Acids Res. 1999, 27, 3035-3041.

27. Shchepinov, M.S.; Udalova, I.A.; Bridgman, A.J.; Southern, E.M. Oligonucleotide dendrimers: synthesis and use as polylabelled DNA probes. Nucleic Acids Res. 1997, 25, 4447-4454.

28. Stears, R.L.; Getts, R.C.; Gullans, S.R. A novel, sensitive detection system for high-density microarrays using dendrimer technology. Physiol. Genomics 2000, 3, 93-99.

29. Oh, K.S.; Kwon, O.; Oh, Y.W.; Sohn, M.J.; Jung, S.; Kim, Y.K.; Kim, M.G.; Rhee, S.K.; Gellissen, G.; Kang, H.A. J. Microbiol. Biotechnol. 2004, 14, 1239-1248.

30. Morris, K.A.; Snir, E.; Pompeia, C.; Koroleva, I.V.; Kachar, B.; Hayashizaki, Y.; Carninci, P.; Soares M.B.; Beisel, K.W. Differential expression of genes within cochlea as defined by custom mouse inner ear microarray. JARO 2005, 6, 75-89.

31. Badiee, A.; Eiken, H.G.; Steen, V.M.; Lovlie, R. Evaluation of five different cDNA labeling methods for microarrays using spike controls. BMC Biotechnology 2003, 3, 23.

32. Romualdi, C.; Trevisian, S.; Celegato, B.; Costa, G.; Lanfranchi, G. Improved detection of differentially expressed genes in microarray experiments through multiple scanning and image integration. Nucleic Acids Res. 2003, 31, e149.

33. Rouse, R.J.D.; Espinoza, C.R.; Niedner, R.H.; Hardiman, G. Development of a microarray assay that measures hybridization stoichiometry in moles. BioTechniques 2004, 36, 464-470.

34. Borucki, M.K.; Reynolds, J.; Call, D.R.; Ward, T.J.; Page, B.; Kadushin, J. Suspension microarray with dendrimer signal amplification allows direct and high-throughput subtyping of Listeria monocytogenes from genomic DNA. J. Clin. Microbiol. 2005, 43, 3255-3259.

(C) 2006 by MDPI (http://www.mdpi.org). Reproduction is permitted for noncommercial purposes. 